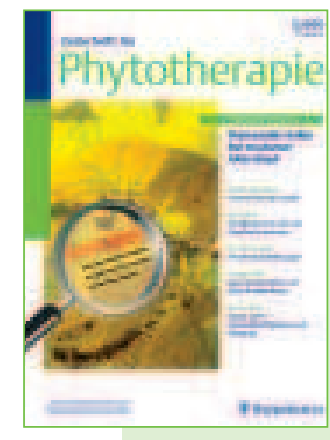

\title{
Liebe Leserinnen und Leser,
}

Sie halten die erste Ausgabe des Jahres 2005 der "Zeitschrift für Phytotherapie» in den Händen und entdecken einiges an Neuem. Die ZPT ändert sich und bleibt sich dabei doch treu. Sie ist und wird auch in Zukunft das deutschsprachige Fachorgan für die Phytotherapie auf wissenschaftlichem Niveau sein. Dennoch war ein Wandel im Sinne von "sich Hinwenden zu den Lesern« notwendig geworden. Dies hat unsere Leserumfrage im letzten Jahr ergeben, und wir möchten an dieser Stelle allen Lesern, die sich daran beteiligt haben, noch einmal herzlich danken.

Fazit der Befragung war, dass die Leser mit der ZPT sehr zufrieden sind, sich aber unter anderem neben kurzgefassten Studienergebnissen auch einen stärkeren Praxisbezug und -nutzen sowie mehr generelle Informationen aus dem Umfeld der Phytotherapie wünschen. Die Arzneipflanzenporträts sind allseits sehr beliebt. Dementsprechend wird die ZPT künftig in vier Hauptrubriken unterteilt: Forschung, Praxis, Forum und Porträt einer Arzneipflanze.

Zeit wird zu einem immer kostbareren Gut und viele Leser wünschen sich, dass wir sie bei der Auswahl aus der Flut an Neuem unterstützen. Dies trifft auch auf die Vielzahl an Studien zu, die im Umfeld der Phytotherapie vorgestellt werden. Herr Professor Schulz hat diese Aufgabe für Sie übernommen. In der neuen Rubrik Klinische Forschung aktuell wird er jene klinischen Studien zur Phytotherapie und den angrenzenden Gebieten sichten, zusammenfassen und für Sie kommentieren, die wichtige Ergebnisse für die tägliche Praxis liefern. So können Sie sich einen schnellen Überblick über interessante Erkenntnisfortschritte verschaffen, ohne selber die vielen Fachzeitschriften daraufhin auswerten zu müssen. Selbstverständlich wird die ZPT auch weiterhin vollständige Forschungsarbeiten und klinische Studien veröffentlichen.

\section{Editorial}

Der gewünschte höhere Praxisbezug wird künftig in den neuen Rubriken Kasuistik und Behandlungsprobleme gewährleistet. Die Informationen sollen jeweils lese- und lerngerecht aufbereitet sowie mit unmittelbaren Umsetzungsempfehlungen und -hilfen für die Praxis verbunden sein.

Eine Zeitschrift, die sich als das deutschsprachige Fachorgan versteht, sollte auch noch einen weiteren Anspruch erfüllen: Sie muss das Forum sein, in dem Sie sich austauschen können und in dem Sie die Informationen zur Phytotherapie finden, die für Sie relevant sind. Dies beinhaltet Themen wie die Gesundheitspolitik (national wie international), Produktinformationen aus der Industrie, Nachrichten aus Verbänden, die Vorstellung einzelner Personen, Kliniken und Institutionen ebenso wie Buchrezensionen oder Kongressberichte. Gerne werden wir auch kontrovers diskutierte Themen in Form von Pro- und Contra-Beiträgen aufnehmen.

Das Porträt einer Arzneipflanze wird auch in Zukunft die Hefte für Sie abrunden.

Wir hoffen sehr, dass Ihnen das geänderte, modernere und leserfreundlichere Layout sowie die neue Konzeption zusagen.

Unser Anspruch ist es, Sie, liebe Leserinnen und Leser auf hohem Niveau über die Phytotherapie zu informieren und Sie dafür zu begeistern. In diesem Sinne freuen wir uns über Anregungen, Kritik und Mithilfe bei der aktiven Gestaltung der künftigen Hefte und wünschen Ihnen viel Freude beim Studium dieser Ausgabe.

Ihre Herausgeber,

Redaktion und Verlag der ZPT 